\title{
Po1-226 Non-referral to Palliative Care:
An Attempt to Investigate Major Reasons
}

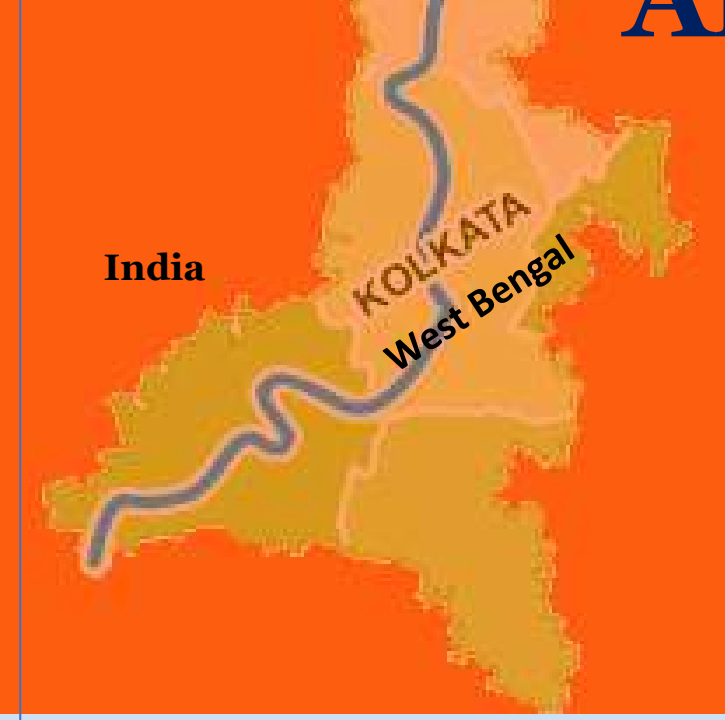

\section{- Research Aim}

To investigate the reasons for non-referral of cancer patients to palliative care by oncologists in the city of Kolkata, India.

\author{
Sanghamitra Bora \\ Palliative Oncology \\ North East Cancer Hospital \& Research Institute \\ Guwahati, Assam, India
}

\section{Background}

Cancer is a huge public health problem in India. Major cancer centers confirm that $2 / 3^{\text {rd }}$ of cancer patients are incurable at presentation and need palliative care. Unequal distribution of services and few palliative care workers continue even after nationwide awareness and steps taken to implement palliative care. Even where services do exist, patients are referred late or not at all. Oncologists care for patients throughout their illness, but little is known of their palliative care referral practices.

\section{Methodology}
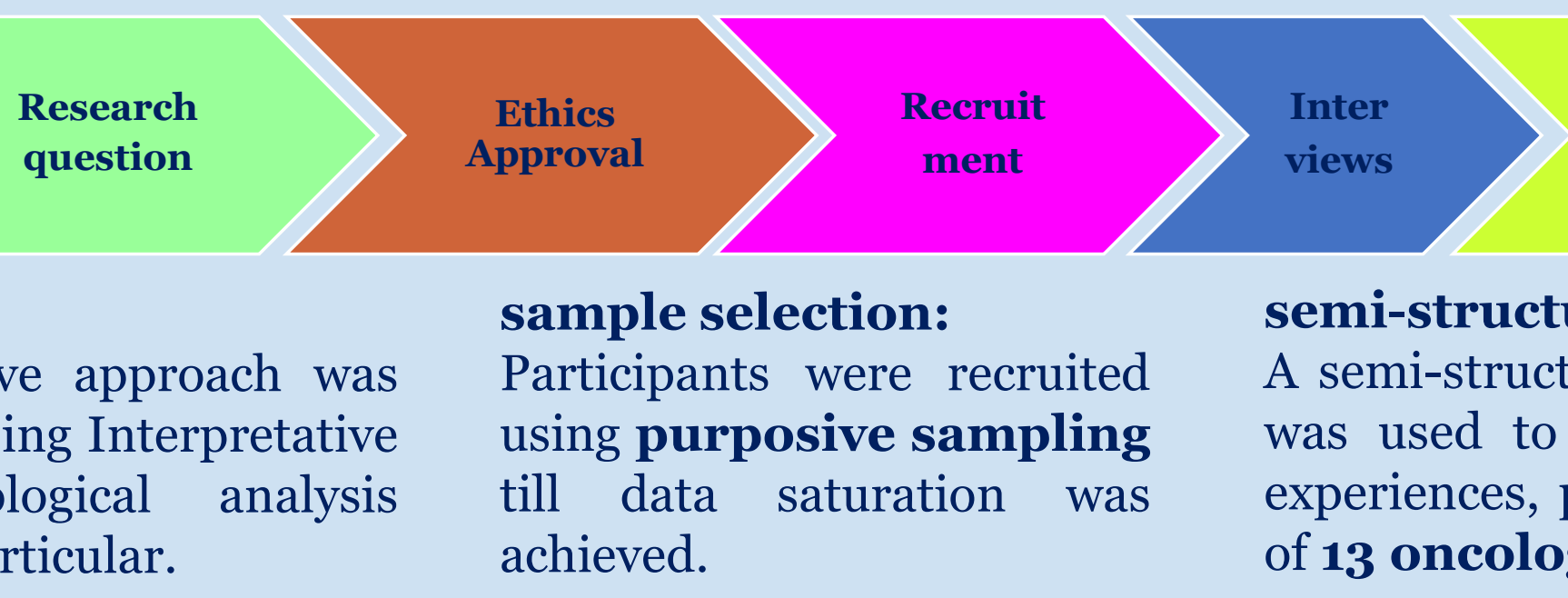

Coding

semi-structured interviews:

A semi-structured interview format was used to explore in-depth the experiences, practices and attitudes of 13 oncologists.

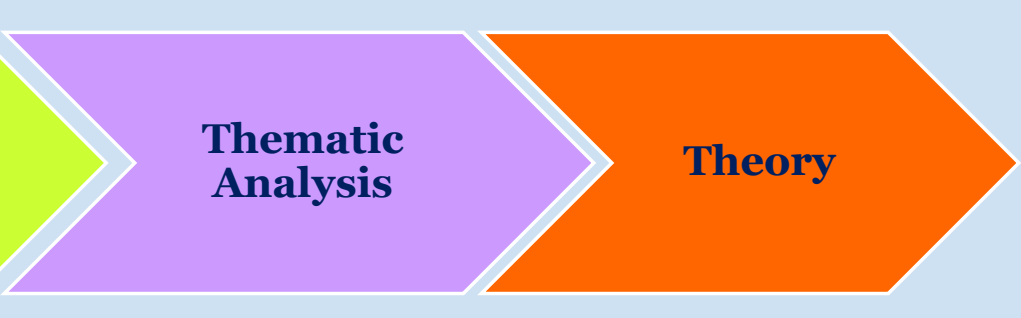

data analysis:

All interviews were transcribed verbatim, coded, \& analysed thematically to form a theory. [IPA] in particular. achieved

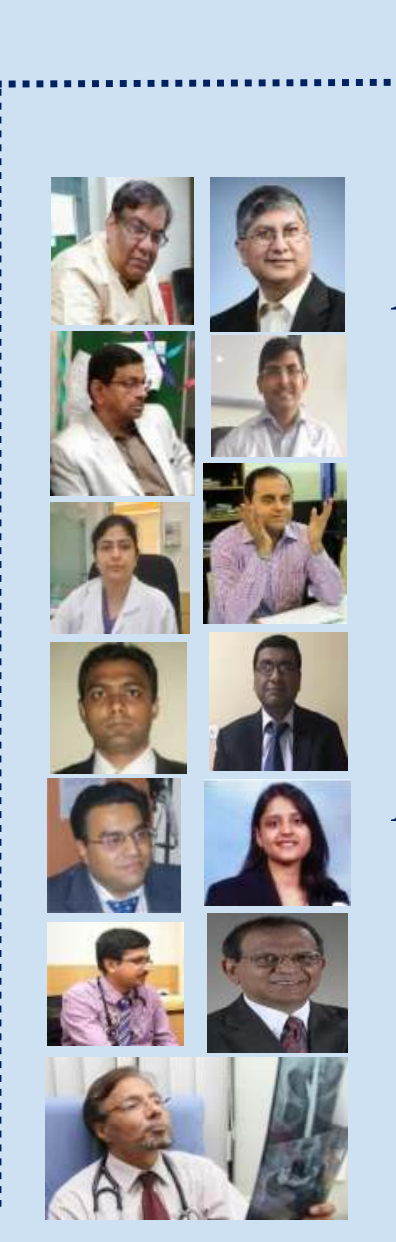

\section{In their own words}

"An oncologist cannot be a pain \& palliative care specialist. All doctors supposedly know part of symptom care but not the whole of it, you know!"

"They (family) force us to keep giving chemos.....it's so hard to convince that they don't take the patient to the ICU. And when they send them to ICU, it gives them a horrendous death."

"I know people who say they do palliative care and admit patients in ICU.......I mean there are people who do that with dying patients!"

"There was actually never a lack of service. It is just that you have named it new, carved out a new niche! That is what you have done!! I have been doing pall care for the last 30 years .... I mean, it's not a new specialty I would say!!”
Lack of proper palliative care service: There oncologists desperately seek support from available resources in any form, whether organizing home care or readmitting the patients in hospital. It reveals their helplessness and frustration over the huge gap in cancer care in their setting.

2) Pressure to continue active treatment: Oncologists are pressurized by patients and family members to continue cancer-directed treatment despite being counselled about possible futility. This is specifically true for young patients and those with insurance. A difference of opinions between oncologists, especially when a $2^{\text {nd }}$ opinion is sought, over continuation of active treatment also contributes to the dilemma.

Lack of awareness among physicians and people: There is a lack of awareness amongst physicians and people regarding benefits of palliative care. Evidence of untrained professionals practicing palliative care, and corporate counterparts looking at this specialty as a non-profitable business are challenges for palliative care to establish its identity in society.

(4) Resistance to accept palliative care as a speciality: Oncologists show resistance in accepting palliative care as a speciality and are ignorant, demonstrate a lack of interest or consider palliative care to be a cost burden, resulting in nonreferral or denial of palliative care for patients.
Primary Insight Oncologists are well intentioned and sensitive towards patient care. An overconfidence of palliative care, that it is the only way a cancer patient is relieved of sufferings, must be diminished to integrate palliative care with mainstream oncology.

\section{Conclusion}

The study builds up on extant evidence on reasons for non-referral to palliative care. Oncologists are well aware of benefits of palliative care, but there lies a gap between the two specialities. Considering the patientoncologist interface to be an important platform for palliative care information sharing, the study emphasizes on awareness generation, education, training, and multidisciplinary team-building efforts, for successful integration of both specialities that is much needed at this juncture of healthcare development.

\section{Opportunities}

There still remains a lot of unanswered questions in the study. These could be explored in more depth with a larger group of oncologists.

More exploration is needed to find the insight of Palliative Care Physicians. 